\title{
Low Rank Co-Diagonal Matrices and Ramsey Graphs
}

\author{
Vince Grolmusz \\ Department of Computer Science \\ Eötvös University, H-1053 Budapest \\ HUNGARY \\ E-mail: grolmusz@cs.elte.hu
}

Submitted: March 7, 2000. Accepted: March 12, 2000

\begin{abstract}
We examine $n \times n$ matrices over $Z_{m}$, with 0 's in the diagonal and nonzeros elsewhere. If $m$ is a prime, then such matrices have large rank (i.e., $n^{1 /(p-1)}-O(1)$ ). If $m$ is a non-prime-power integer, then we show that their rank can be much smaller. For $m=6$ we construct a matrix of $\operatorname{rank} \exp (c \sqrt{\log n \log \log n})$. We also show, that explicit constructions of such low rank matrices imply explicit constructions of Ramsey graphs.
\end{abstract}

Keywords: composite modulus, explicit Ramsey-graph constructions, matrices over rings, co-diagonal matrices

\section{Introduction}

In this work we examine matrices over a ring $R$, such that the diagonal elements of the matrix are all 0's, but the elements off the diagonal are not zero (we shall call these matrices co-diagonal over $R$ ). We define the rank of a matrix over a ring, and show that low rank codiagonal matrices over $Z_{6}$ naturally correspond to graphs with small homogenous vertex sets (i.e., cliques and anti-cliques). Consequently, explicitly constructible low rank co-diagonal matrices over $Z_{6}$ imply explicit Ramsey graph constructions. Our best construction reproduces the logarithmic order of magnitude of the Ramsey-graph of Frankl and Wilson [5], continuing the sequence of results on new explicit Ramsey graph constructions of Alon [1] and Grolmusz [6]. Our present result, analogously to the constructions of [6] and [1], can be generalized to more than one color. 
Our results give a recipe for constructing explicit Ramsey graphs from explicit low rank co-diagonal matrices over $Z_{6}$, analogously to the way that our results gave a method for constructing explicit Ramsey graphs from certain low degree polynomials over $Z_{6}$ in [6]. In this sense, our results may lead to improved Ramsey graph constructions, if lower rank co-diagonal matrix constructions exist.

Definition 1 Let $R$ be a ring and let $n$ be a positive integer. We say, that $n \times n$ matrix $A=\left\{a_{i j}\right\}$ is a co-diagonal matrix over $R$, if $a_{i j} \in R, i, j=1,2, \ldots, n$ and $a_{i i}=0, a_{i j} \neq 0$, for all $i, j=1,2, \ldots, n, i \neq j$.

We say, that $A$ is an upper co-triangle matrix over $R$, if $a_{i j} \in R, i, j=1,2, \ldots, n$ and $a_{i i}=0, a_{i j} \neq 0$, for all $1 \leq i<j \leq n$. $A$ is a lower co-triangle matrix over $R$, if $a_{i j} \in R, i, j=1,2, \ldots, n$ and $a_{i i}=0, a_{i j} \neq 0$, for all $1 \leq j<i \leq n$. A matrix is co-triangle, if it is either lower-or upper co-triangle.

We will also need the definition of the rank of a matrix with elements in a ring. The following definition is a generalization of the matrix rank over fields to matrices over rings:

Definition 2 Let $R$ be a ring and let $n$ be a positive integer. We say, that $n \times n$ matrix $A$ over $R$ has rank 0 if all of the elements of $A$ are 0 . Otherwise, the rank over the ring $R$ of matrix $A$ is the smallest $r$, such that $A$ can be written as

$$
A=B C
$$

over $R$, where $B$ is an $n \times r$ and $C$ is an $r \times n$ matrix. The rank of $A$ over $R$ is denoted by $\operatorname{rank}_{R}(A)$.

It is easy to see, that this definition of the matrix rank coincides with the usual matrixrank over $R$, when $R$ is a field. The following property of the usual matrix rank also holds:

Lemma 3 Let $R$ be a ring and let $A$ and $A^{\prime}$ be two $n \times n$ matrices. Then $\operatorname{rank}_{R}\left(A+A^{\prime}\right) \leq$ $\operatorname{rank}_{R}(A)+\operatorname{rank}_{R}\left(A^{\prime}\right)$.

Proof: $\quad$ Let $A=B C$ and $A^{\prime}=B^{\prime} C^{\prime}$, where $B$ is an $n \times r$ and $C$ is an $r \times n$ matrix, while $B^{\prime}$ is an $n \times r^{\prime}$ and $C^{\prime}$ is an $r^{\prime} \times n$ matrix. Then $A+A^{\prime}$ can be given as $B^{\prime \prime} C^{\prime \prime}$, where $B^{\prime \prime}$ is an $n \times\left(r+r^{\prime}\right)$ matrix, formed from the union of the columns of $B$ and $B^{\prime}$, and $C^{\prime \prime}$ is an $\left(r+r^{\prime}\right) \times n$ matrix, formed from the union of rows of $C$ and $C^{\prime}$.

The following theorem shows, that for any prime $p$, the co-triangle (and, consequently, the co-diagonal) matrices over the $p$-element field have large rank:

Theorem 4 Let $p$ be a prime, and let $A$ be an $n \times n$ co-triangle matrix over $G F_{p}$. Then

$$
\operatorname{rank}_{\mathrm{GF}_{p}}(A) \geq n^{1 /(p-1)}-p .
$$


Proof: We may assume that $A$ is a lower co-triangle matrix. Let $r=\operatorname{rank}_{\mathrm{GF}_{p}}(A)$, and let $B=\left\{b_{i j}\right\}$ be an $n \times r, C=\left\{c_{i j}\right\}$ be an $r \times n$ matrix over $\mathrm{GF}_{p}$, such that:

$$
A=B C \text {. }
$$

For $i=1,2, \ldots, n$ let us consider the following polynomials:

$$
P_{i}\left(x_{1}, x_{2}, \ldots, x_{r}\right)=\sum_{k=1}^{r} b_{i k} x_{j} .
$$

From (1),

$$
P_{i}\left(c_{1 j}, c_{2 j}, \ldots, c_{r j}\right)=\left\{\begin{array}{l}
0, \text { if } i=j \\
\neq 0, \text { if } i>j .
\end{array}\right.
$$

Consequently, by the triangle criterion [2], polynomials

$$
Q_{i}\left(x_{1}, x_{2}, \ldots, x_{r}\right)=1-P_{i}^{p-1}\left(x_{1}, x_{2}, \ldots, x_{r}\right),
$$

for $i=1,2, \ldots, n$, form a linearly independent set in the vector space of dimension

$$
\left(\begin{array}{c}
r+p-2 \\
p-1
\end{array}\right)+1
$$

of polynomials of form $Q+\alpha$, where $Q$ is an $r$-variable homogeneous polynomial of degree $p-1$ and $\alpha \in \mathrm{GF}_{p}$. (To prove this without the triangle criterion of [2], one should observe that $Q_{k}$ is zero on column $i$ of matrix $C$ for $i<k$, and it is 1 for column $k$ of $C$; so $Q_{i}$ cannot be given as a linear combination of some $Q_{k_{j}}$ 's, each $k_{j}>i$.) Consequently,

$$
n \leq\left(\begin{array}{c}
r+p-2 \\
p-1
\end{array}\right)+1 \leq(r+p)^{p-1} .
$$

We are interested in the following question:

Question. Let $R=Z_{m}$, what is the minimum rank of an $n \times n$ co-triangle (or co-diagonal) matrix over $R$ ?

If $m=p$ a prime, then by Theorem 4 we have that the rank should be at least $n^{1 / p-1}-p$. What can we say for non-prime $m$ 's?

The main motivation of this question is the following theorem:

Theorem 5 Let $A=\left\{a_{i j}\right\}$ be an $n \times n$ co-triangle matrix over $R=Z_{6}$, with $r=\operatorname{rank}_{Z_{6}}(A)$. Then there exists an n-vertex graph $G$, containing neither a clique of size $r+2$ nor an anti-clique of size

$$
\left(\begin{array}{c}
r+1 \\
2
\end{array}\right)+2
$$


Proof: $\quad$ Suppose, that $A$ is a lower co-triangle matrix. If the $Z_{6}$ rank of $A$ is $r$, then both the $\mathrm{GF}_{2}$ and $\mathrm{GF}_{3}$ ranks of $A$ are at most $r$. Let $V=\left\{v_{1}, v_{2}, \ldots, v_{n}\right\}$. For any $i>j$, let us connect $v_{i}$ and $v_{j}$ with an edge, if $a_{i j}$ is odd. Then any clique of size $t$ will correspond to a $t \times t$ lower co-triangle minor over $\mathrm{GF}_{2}$, so from (3),

$$
t \leq r+1
$$

Any anti-clique of size $t$ will correspond to a $t \times t$ lower co-triangle minor over $\mathrm{GF}_{3}$, so from $(3)$,

$$
t \leq\left(\begin{array}{c}
r+1 \\
2
\end{array}\right)+1
$$

From Theorem 5 one can get a lower bound for the rank, using estimations for the Ramsey numbers. Our original bound was significantly improved by Noga Alon, who allowed us to include his proof here.

Theorem 6 Let $A=\left\{a_{i j}\right\}$ be an $n \times n$ co-triangle matrix over $R=Z_{6}$. Then

$$
\operatorname{rank}_{Z_{6}}(A) \geq \frac{\log n}{2 \log \log n}-2
$$

Proof: By the result of Ramsey [7] and Erdös and Szekeres [4], every $n$-vertex graph has either a clique on $k$, or an anti-clique on $\ell$ vertices, if

$$
n \geq\left(\begin{array}{c}
k+\ell-2 \\
k-1
\end{array}\right) \text {. }
$$

If we set $k=\left\lfloor\frac{1}{2} \frac{\log n}{\log \log n}\right\rfloor$, and $\ell=\left\lfloor\log ^{2} n\right\rfloor$, then we get from Theorem 5 , that both $r+2 \leq k$ and $\left(\begin{array}{c}r+1 \\ 2\end{array}\right)+2 \leq \ell$ cannot be satisfied, and this completes the proof.

The proof of Theorem 5 also proves

Theorem 7 Suppose, that there exists an explicitly constructible $n \times n$ co-triangle matrix $A=\left\{a_{i j}\right\}$ over $R=Z_{6}$, with $r=\operatorname{rank}_{Z_{6}}(A)$. Then one can explicitly construct an $n$-vertex Ramsey-graph, without homogenous vertex-sets of size

$$
\left(\begin{array}{c}
r+1 \\
2
\end{array}\right)+2 .
$$

Our main result is that there do exist explicitly constructible low-rank co-diagonal matrices over $Z_{6}$, implying explicit Ramsey-graph constructions. 
Theorem 8 There exists a $c>0$ such that for all positive integer $n$, there exists an explicitly constructible $n \times n$ co-diagonal matrix $A=\left\{a_{i j}\right\}$ over $R=Z_{6}$, with

$$
\operatorname{rank}_{Z_{6}}(A) \leq 2^{c \sqrt{\log n \log \log n}} .
$$

Theorem 8 together with Theorem 5 , gives an explicit Ramsey-graph construction on $n$ vertices, without a homogeneous vertex-set of size $2^{c^{\prime}} \sqrt{\log n \log \log n}$, for some $c^{\prime}>0$, or in other words, an explicit Ramsey-graph construction on

$$
2^{\frac{c^{\prime \prime} \log ^{2} t}{\log \log t}}
$$

vertices, without homogeneous vertex-set of size $t$, for some $c^{\prime \prime}>0$. This bound was first proven by Frankl and Wilson [5] with a larger (better) constant than our $c^{\prime \prime}$, using the famous Frankl-Wilson theorem [5]. We also gave a construction, using the BBR polynomial [3] and also the Frankl-Wilson theorem in [6].

A generalization of our main result for ring $Z_{m}$, where $m$ has more than two prime divisors:

Theorem 9 For any $m=p_{1}^{\alpha_{1}} p_{2}^{\alpha_{2}} \ldots p_{\ell}^{\alpha_{\ell}}$, where the $p_{i}$ 's are distinct primes, there exists a $c=c_{m}>0$ such that for all positive integer $n$, there exists an explicitly constructible $n \times n$ co-diagonal matrix $A=\left\{a_{i j}\right\}$ over $R=Z_{m}$, with

$$
\operatorname{rank}_{Z_{m}}(A) \leq 2^{c \sqrt[\ell]{\log n(\log \log n)^{\ell-1}}}
$$

\section{Constructing Low Rank mod 6 Co-Diagonal Matri- ces}

In this section we prove Theorems 8 and 9 .

Our main tool is the following theorem (choosing $m=6$ and $\ell=2$ ):

Theorem 10 (Barrington, Beigel, Rudich[3]) Given $m=p_{1}^{\alpha_{1}} p_{2}^{\alpha_{2}} \ldots p_{\ell}^{\alpha_{\ell}}$ where the $p_{i}$ are distinct primes, then there exists an explicitly constructible multi-linear polynomial $P$ with integer coefficients, with $k$ variables, and of degree $O\left(k^{1 / \ell}\right)$ which satisfies for $x \in\{0,1\}^{k}$, that $P(x)=0$ over $Z_{m}$ iff $x=(0,0, \ldots, 0)$.

Let $k$ be the smallest integer such that $n \leq k^{k}$. Let $B=\{0,1,2, \ldots, k-1\}$. Let us define $\delta: B \times B \rightarrow\{0,1\}$ as follows:

$$
\delta(u, v)=\left\{\begin{array}{l}
1, \text { if } u=v \\
0 \text { otherwise }
\end{array}\right.
$$


Then matrix $\bar{A}$ is defined as follows: both the rows and the columns of $\bar{A}$ correspond to the elements of the set $B^{k}$. The entry of matrix $\bar{A}$ in the intersection of a row, corresponding to $u=\left(u_{1}, u_{2}, \ldots, u_{k}\right) \in B^{k}$ and of a column, corresponding to $v=\left(v_{1}, v_{2}, \ldots, v_{k}\right) \in B^{k}$ is the number:

$$
P\left(1-\delta\left(u_{1}, v_{1}\right), 1-\delta\left(u_{2}, v_{2}\right), \ldots, 1-\delta\left(u_{k}, v_{k}\right)\right) .
$$

If $u=v$, then all of the $\delta\left(u_{i}, v_{i}\right)$ 's are 1 , so the value of $P$ is 0 . So the diagonal of $\bar{A}$ is all-0, but no other elements of the matrix are 0 over $Z_{6}$, consequently, $\bar{A}$ is co-diagonal over $Z_{6}$.

Multi-linear polynomial $P$ has degree $O(\sqrt{k})$, so $(5)$ can be written as the sum of

$$
\left(\begin{array}{c}
k \\
\leq c\lfloor\sqrt{k}\rfloor
\end{array}\right)=\sum_{i=0}^{c\lfloor\sqrt{k}\rfloor}\left(\begin{array}{l}
k \\
i
\end{array}\right)<k^{c \sqrt{k}}
$$

monomials of the form:

$$
a_{i 1, i 2, \ldots, i s} \delta\left(u_{i 1}, v_{i 1}\right) \delta\left(u_{i 2}, v_{i 2}\right), \ldots \delta\left(u_{i s}, v_{i s}\right)
$$

where $c$ is positive, (in fact, $c<3$ is also satisfied), $a_{i 1, i 2, \ldots, i s}$ is an integer between 0 and 5 , and $s \leq c \sqrt{k}$.

Since the $(u, v)$ entry of $\bar{A}$ is the value (5), and (5) can be written as the sum of monomials in (7), matrix $\bar{A}$ can be written as the sum of matrices $D_{i 1, i 2, \ldots, i s}$, where the entry of matrix $D_{i 1, i 2, \ldots, i s}$ in the intersection of a row, corresponding to $u=\left(u_{1}, u_{2}, \ldots, u_{k}\right) \in B^{k}$ and of a column, corresponding to $v=\left(v_{1}, v_{2}, \ldots, v_{k}\right) \in B^{k}$ is equal to the value of (7).

It is easy to verify that $D_{i 1, i 2, \ldots, i s}$ can be written into the following form (applying the same, suitable permutation to the rows and columns):

$$
D_{i 1, i 2, \ldots, i s}=a_{i 1, i 2, \ldots, i s}\left(\begin{array}{ccccccccccccc}
1 & 1 & 1 & 1 & 0 & 0 & 0 & 0 & \ldots & 0 & 0 & 0 & 0 \\
1 & 1 & 1 & 1 & 0 & 0 & 0 & 0 & \ldots & 0 & 0 & 0 & 0 \\
1 & 1 & 1 & 1 & 0 & 0 & 0 & 0 & \ldots & 0 & 0 & 0 & 0 \\
1 & 1 & 1 & 1 & 0 & 0 & 0 & 0 & \ldots & 0 & 0 & 0 & 0 \\
0 & 0 & 0 & 0 & 1 & 1 & 1 & 1 & \ldots & 0 & 0 & 0 & 0 \\
0 & 0 & 0 & 0 & 1 & 1 & 1 & 1 & \ldots & 0 & 0 & 0 & 0 \\
0 & 0 & 0 & 0 & 1 & 1 & 1 & 1 & \ldots & 0 & 0 & 0 & 0 \\
0 & 0 & 0 & 0 & 1 & 1 & 1 & 1 & \ldots & 0 & 0 & 0 & 0 \\
\vdots & \vdots & \vdots & \vdots & \vdots & \vdots & \vdots & \vdots & \ddots & \vdots & \vdots & \vdots & \vdots \\
0 & 0 & 0 & 0 & 0 & 0 & 0 & 0 & \ldots & 1 & 1 & 1 & 1 \\
0 & 0 & 0 & 0 & 0 & 0 & 0 & 0 & \ldots & 1 & 1 & 1 & 1 \\
0 & 0 & 0 & 0 & 0 & 0 & 0 & 0 & \ldots & 1 & 1 & 1 & 1 \\
0 & 0 & 0 & 0 & 0 & 0 & 0 & 0 & \ldots & 1 & 1 & 1 & 1
\end{array}\right)
$$


Let us observe, that the number of all-1 square minors, covering the diagonal is $k^{s}$. Then, from Lemma 3 the rank of $D_{i 1, i 2, \ldots, i s}$ is $k^{s}, s \leq c \sqrt{k}$. It follows from this and from (6), that the rank of $\bar{A}$ is at most $k^{2 c \sqrt{k}}$.

Let matrix $A$ be defined as the $n \times n$ upper left minor of matrix $\bar{A}$. Obviously, $A$ is also a co-diagonal matrix, and its rank is at most $k^{2 c \sqrt{k}}$. Due to the choice of $k$ the statement follows.

The proof of Theorem 9 follows the same steps as the proof of Theorem 8 . If $m$ has $\ell$ prime divisors, then polynomial $P$ has degree $O\left(k^{1 / \ell}\right)$, so matrix $D_{i 1, i 2, \ldots, i s}$ has rank at most $k^{s}, s \leq c k^{1 / \ell}$, and co-diagonal matrix $A$ has rank at most $k^{2 c k^{1 / \ell}}$, and this proves the theorem.

Acknowledgment. The author is grateful to Noga Alon for his comments and for his significant improvement of Theorem 6, and to Laci Babai for the discussions on this topic .

\section{References}

[1] N. Alon. The Shannon capacity of a union. Combinatorica, 18:301-310, 1998.

[2] L. Babai and P. Frankl. Linear algebra methods in combinatorics. Department of Computer Science, The University of Chicago, September 1992. preliminary version.

[3] D. A. M. Barrington, R. Beigel, and S. Rudich. Representing Boolean functions as polynomials modulo composite numbers. Comput. Complexity, 4:367-382, 1994. Appeared also in Proc. 24th Ann. ACM Symp. Theor. Comput., 1992.

[4] P. Erdős and G. Szekeres. A combinatorial problem in geometry. Composition Math., 2:464-470, 1935.

[5] P. Frankl and R. M. Wilson. Intersection theorems with geometric consequences. Combinatorica, 1(4):357-368, 1981.

[6] V. Grolmusz. Superpolynomial size set systems with restricted intersections mod 6 and explicit Ramsey graphs. Combinatorica, 20:1-14, 2000. Conference version appeared in Proc. COCOON'97, LNCS 1276.

[7] F. P. Ramsey. On a problem of formal logic. Proc. London Math. Soc., 30:264-286, 1930. 